\title{
A PLETHYSMOGRAPHIC METHOD FOR THE QUANTITATIVE MEASUREMENT OF THE BLOOD FLOW IN THE. FOOT ${ }^{1}$
}

\author{
By EUGENE A. STEAD, JR., AND PAUL KUNKEL \\ (From the Thorndike Memorial Laboratory, Second and Fourth Medical Services (Harvard), \\ Boston City Hospital, and the Department of Medicine, \\ Harvard Medical School, Boston)
}

(Received for publication June 10, 1938)

The importance of determining the blood flow in the foot in the study of vascular diseases involving the lower extremities is well recognized. Various methods, such as measurements of skin temperature, oscillometric tracings, the response to arterial occlusion, the histamine test, and tracings from toe plethysmographs, have been used to determine the functional capacity of the blood vessels of the foot. All these methods, however, yield only indirect indices of the total blood flow. The purpose of this investigation was to devise a method for the quantitative measurement of the blood flow in the foot in health and in disease. A plethysmograph has been designed which is based on the principle of Hewlett and Van Zwaluwenburg (1). The instrument is similar to that employed by Freeman (2) for measuring the blood flow in the hand. When the venous outflow is occluded by a "collecting pressure" lower than the diastolic pressure, the rate of initial increase in the foot volume is a measure of the amount of blood flowing to the foot.

Figure 1 shows the general design of the instrument. It is constructed of rigid brass sheets soldered together. The front presents an opening $15 \mathrm{~cm}$. in diameter, through which the foot is inserted. A wire grid supports the foot 2.5 $\mathrm{cm}$. from the sides and the floor of the instrument. On the upper surface are openings for a thermometer and for a rubber tube which is connected with a Brodie bellows of $10 \mathrm{cc}$. capacity. Figure 2 is a sagittal section drawn to scale. The water is heated by two cartridge units of 100 and 200 watts capacity, respectively. The heaters are enclosed in jackets tooled of solid brass. This precaution is necessary as soldered seams may leak and cause a short circuit in the

1 The expenses of this investigation were defrayed in part by a grant from the Proctor Fund of Harvard University for the study of chronic diseases. heating unit. The jackets for the heaters are soldered into the plethysmograph beneath the grid and parallel to the back surface in such a position that they do not project beneath the heel where local heat may cause discomfort. A propeller for stirring the water is inserted through the back wall near its base. This is rotated by a shaft from a universal motor of 5000 r.p.m. with a gear ratio of 35 to 1 . The electrical conductivity of the shaft is interrupted by a fiber joint. When two or more plethysmographs are employed, they are connected by a copper wire soldered to the instruments and grounded.

The foot is inserted to the level of the malleoli through a thin rubber membrane having a cuff which fits lightly enough not to constrict the superficial veins and which is attached to the foot with rubber cement. The plethysmograph is placed at heart level. With the subject recumbent, and the calf well supported in order to minimize pressure on the heel, the foot is inserted into the instrument and rests on a rubber pad. The rubber membrane is then stretched over the flanged opening of the instrument which has previously been coated with rubber cement and held in place by a metal ring making a watertight joint. A felt pad one-half inch thick is adjusted to the ankle and is held rigid by an iris diaphragm of three brass plates which are secured by right-angle clamps and wing nuts. The plethysmograph is filled with water at the desired temperature and $60 \mathrm{cc}$. are then aspirated to allow for air transmission to the Brodie bellows. No determinations are made until the water bath has been at a constant temperature for 30 minutes. A pressure cuff $4 \mathrm{~cm}$. in width is applied to the leg just proximal to the plethysmograph. This cuff may be inflated very rapidly from a 20-liter bottle fitted with a pressure gauge. In applying the rubber membrane, the felt pad, 




Fig. 1. Plethysmograph with Foot in Place

(1) Iris diaphragm; (2) right-angle clamp and wing nut for securing iris diaphragm; (3) outlet for thermometer; (4) outlet for rubber tube to Brodie bellows; (5) cartridge unit heater (partially inserted); (6) felt pad; (7) rubber membrane; (8) pressure cuff.

and the pressure cuff, care is taken not to cause an elevation of the venous pressure. When sudden pressure is applied in the cuff a smooth, rising curve is traced on the smoked drum, the slope of which represents the rate at which the blood is flowing into the foot.

In practice, the system is calibrated on a slowly revolving drum by adding $5 \mathrm{cc}$. of air directly to the rubber tube which connects the instrument with the recording bellows. The tube is clamped at the plethysmograph. This method gives a greater deflection of the recording lever than is obtained when air is displaced by increasing the volume of water in the plethysmograph. This introduces one source of error into the determinations, since during the recording of the blood flow, air is displaced into the recording system by the increase in the volume of the foot. A second source of error results from the inertia of the plethysmograph-bellows system. This is not apparent during the calibration on a slow drum, but it becomes obvious with the more rapid changes in volume associated with the actual determinations of blood flow. In order to establish the correction factor for these two sources of error, six experiments were done with the foot in place. The water in the plethysmograph was kept between 17 and $20^{\circ} \mathrm{C}$. in order to minimize the spontaneous changes in foot volume. In these experiments water was added to the instrument at a known rate by a constant injection apparatus. The tracings were recorded in the usual 


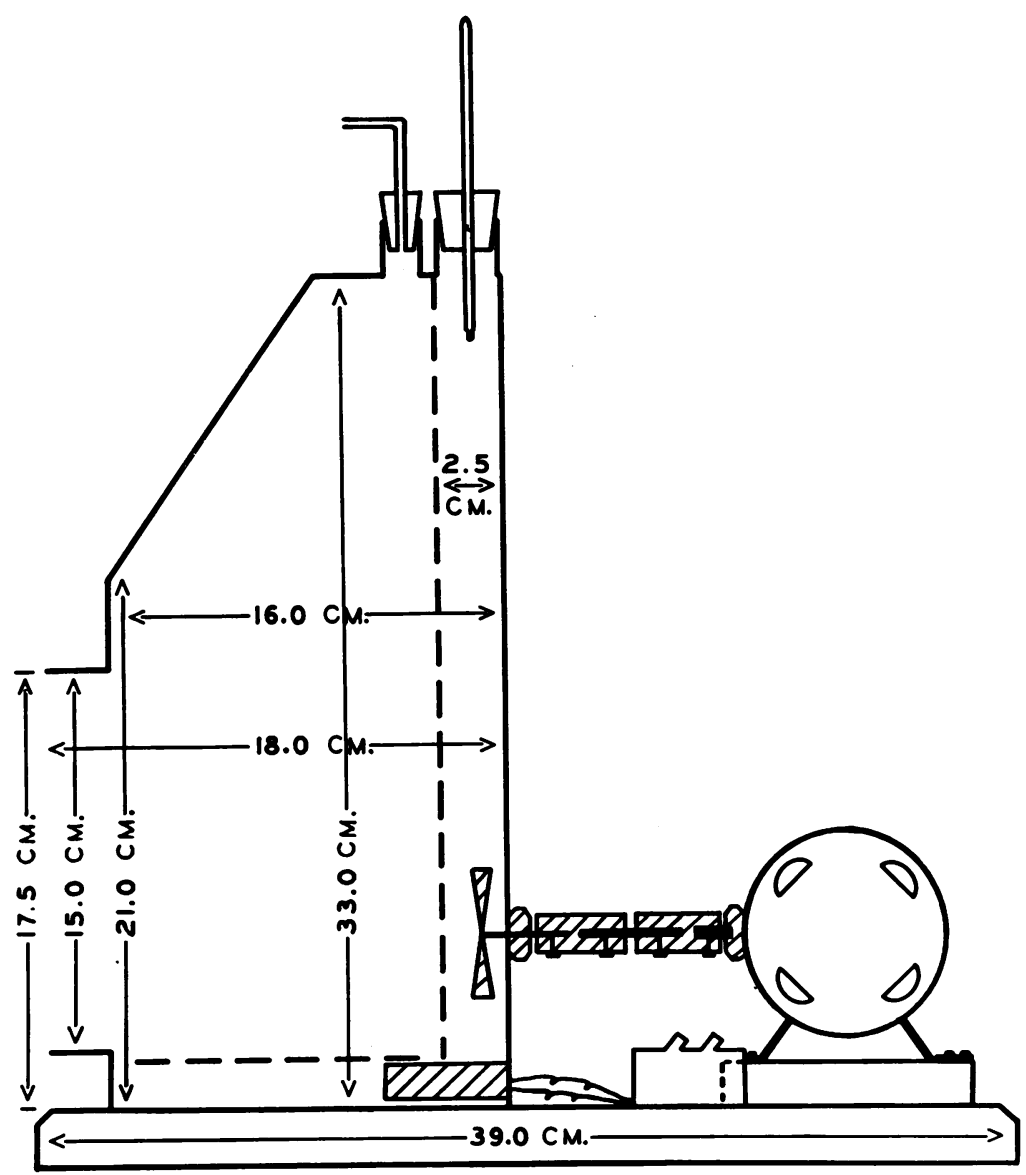

Fig. 2. Sagittal Section Drawn to Scale

manner. The amount of water added in unit time was calculated by the usual method of calibration and was compared with the amount of water actually added. It was found that the actual increase in the volume of water was from 10 per cent to 16 per cent, or an average of 13 per cent, greater than the increase calculated from an average of five tracings in each experiment. Therefore, in order to compensate for these two errors, 13 per cent has been added to the flows as calculated from the air calibration. With the foregoing correction, these figures show an actual instrumental variation of \pm 3 per cent when the flows are calculated as an average of five determinations. The error of the method cannot be determined from the amount of variation between the individual blood flow curves since the variations resulting from changes in vasomotor tone are considerably greater than the error of the instrument.
Figure 3 shows a blood flow tracing with the lines drawn for the calculation. After projecting the slope of the curve to the base line, the number of seconds required for the curve to rise a distance equivalent to $3 \mathrm{cc}$. is determined (this height is obtained by the introduction of $3 \mathrm{cc}$. of air into the recording system). The volume of the foot in cubic centimeters is determined by subtracting from the volume of the instrument the amount of water necessary to fill the plethysmograph when the foot is in place. To reduce the blood flow to cubic centimeters per minute per $100 \mathrm{cc}$. of foot the following formula is used:

$$
\text { Blood flow }=\frac{H 60}{S V} \times 1.13 \text {. }
$$

$H$ is the volume increase in cubic centimeters in $S$ seconds. $V$ is the volume of foot in $100 \mathrm{cc}$. or fraction thereof. Substituting the values in Figure 3, we have: 


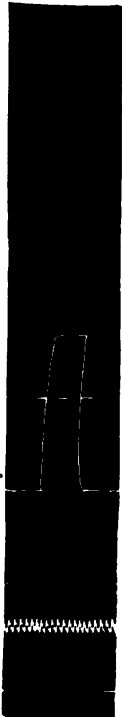

A

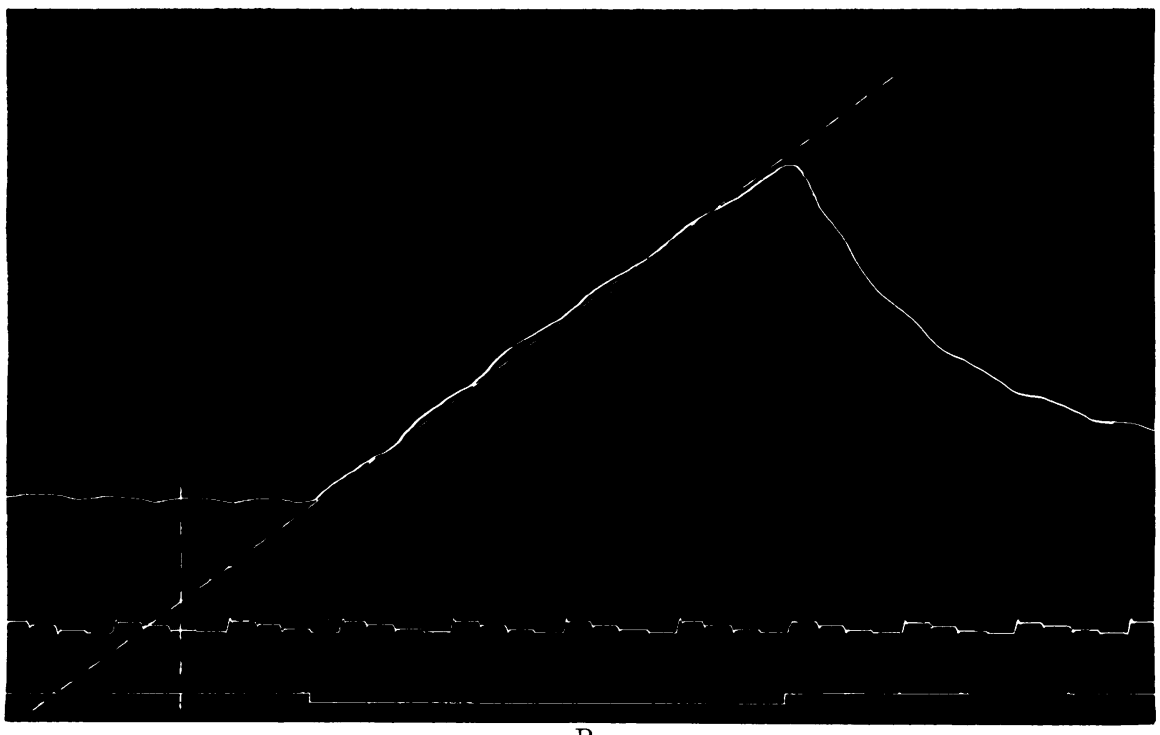

$\mathrm{B}$

FI(;. 3

1. Calibration on slowly revolving drum by adding 5 cc. of air to bellows. Horizontal broken line at 3 cc. mark. B. Typical blood flow curve with broken line continuing slope of curve to base line and interrupted at a distance equivalent to $3 \mathrm{cc}$ obtained from calibration (Figure 3.1). Drop in base line indicates period during which cuff pressure was applied.

Blood flow $=\frac{3 \times 60}{1.12 \times 10.10} \times 1.13=18 \mathrm{cc}$. per minute per 100 cc. of foot.

An attempt was macle to determine whether the $4 \mathrm{~cm}$. cuff at the ankle is adequate to block the venous return from the foot completely at the moment the pressure is applied. When the $+\mathrm{cm}$. cuff was replaced either by one $12 \mathrm{~cm}$. cuff. or by two $12 \mathrm{~cm}$. cuffs applied about the midcalf and the lower thigh, respectively, no increase in flow was obtained over that observed with the $4 \mathrm{~cm}$. cuff.

In normal persons, accurate curves are obtained with occluding pressures as high as the brachial diastolic pressure. In the presence of obliterative vascular disease, however, care must be taken that the occluding pressure does not prevent the inflow of the arterial blood. This may happen before the collecting pressure is raised to the brachial diastolic level. In these cases the collecting pressure that gives the greatest constant increase in foot volume is used. The ankle cuff is carefully adjusted so as not to clisplace the foot in the plethysmograph when the pressure is applied. At times some displacement cannot be avoided, and in such cases the first part of the slope is disregarded.

The instrument is sensitive enough to record pulse waves, respiratory waves, and changes in volume caused by variations in vasomotor tone. Thus, by using a drum at slow speed the vasomotor reactions of the foot can be studied in detail.

\section{SLMMIRY}

A plethysmographic method has been clescribed for the quantitative measurement of the blood flow in the foot. With a stanclard correction for the inertia of the plethysmograph-bellows system, the instrumental error was found to be \pm 3 per cent. The plethysmograph is also useful in the study of the vasomotor reactions of the vessels of the foot.

\section{BIBLIOGRAPHY}

1. Hewlet1, A. W., and Van Zwaluwenburg. I. G., The rate of blood flow in the arm. Heart, 1909-10, $1,87$.

2. Freeman, N. E., The effect of temperature on the rate of blood flow in the normal and in the sympathectomized hand. Am. J. Physiol., 1935, 113, 384. 